\title{
Eigenfunctions and fundamental solutions of the Caputo fractional Laplace and Dirac operators*
}

\author{
M. Ferreira ${ }^{\dagger, \ddagger}, \quad$ N. Vieira ${ }^{\ddagger}$ \\ † School of Technology and Management, \\ Polytechnic Institute of Leiria \\ P-2411-901, Leiria, Portugal. \\ E-mail: milton.ferreira@ipleiria.pt \\ $\ddagger$ CIDMA - Center for Research and Development in Mathematics and Applications \\ Department of Mathematics, University of Aveiro \\ Campus Universitário de Santiago, 3810-193 Aveiro, Portugal. \\ E-mail: mferreira@ua.pt,nloureirovieira@gmail.com
}

\begin{abstract}
In this paper, by using the method of separation of variables, we obtain eigenfunctions and fundamental solutions for the three parameter fractional Laplace operator defined via fractional Caputo derivatives. The solutions are expressed using the Mittag-Leffler function and we show some graphical representations for some parameters. A family of fundamental solutions of the corresponding fractional Dirac operator is also obtained. Particular cases are considered in both cases.
\end{abstract}

Keywords: Fractional partial differential equations; Fractional Laplace and Dirac operators; Caputo derivative; Eigenfunctions; Fundamental solution.

MSC 2010: 35R11; 35A08; 26A33; 30G35; 35C05.

\section{Introduction}

In the last decades the interest in fractional calculus increased substantially. Among all the subjects there is a considerable interest in the study of ordinary and partial fractional differential equations regarding the mathematical aspects and methods of their solutions, and their applications in diverse areas such as physics, chemistry, engineering, optics or quantum mechanics (see, for example $[7-12,14,16]$ ).

Here we consider a fractional Laplace operator in 3-dimensional space using Caputo derivatives with different order of differentiation for each direction. Previous approaches for this type of operators where considered in [15], [3], and [4]. In [15] the author studied eigenfunctions and fundamental solutions for the two-parameter fractional Laplace operator defined with Riemann-Liouville fractional derivatives. In [3] the authors extended the results for three dimensions and derived also fundamental solutions for the fractional Dirac operator which factorizes the fractional Laplace operator. Since there is a duality relation between Caputo and RiemannLiouville fractional derivatives presented in the formula of fractional integration by parts, there is need to study also fractional Laplace and Dirac operators with fractional derivatives defined in the Caputo sense. The aim of this paper is to use the method of separation of variables to present a formula for the family of eigenfunctions and fundamental solutions of the three-parameter fractional Laplace operator defined by Caputo fractional derivatives, as well as a family of fundamental solutions of the associated fractional Dirac operator. For the

*The final version is published in Modern trends in Hypercomplex Analysis, Modern Trends in Hypercomplex Analysis, Trends in Mathematics Series, S. Bernstein, U. Khler, I. Sabadini, F.Sommen (Eds.), Birkhäuser, Basel, (2016), 191-202. It as available via the website http://www.springer.com/us/book/9783319425283 
sake of simplicity we restrict ourselves to the three dimensional case, however the results can be generalized for an arbitrary dimension. We observe that these operators were considered in [4] where the authors applied an operational approach via Laplace transform to construct general families of eigenfunctions and fundamental solutions.

The structure of the paper reads as follows: in the Preliminaries we recall some basic facts about fractional calculus, special functions and Clifford analysis, which are necessary for the development of this work. In Subsection 3.1 we use the method of separation of variables to describe a complete family of eigenfunctions and fundamental solutions of the fractional Laplace operator. In Subsection 3.2 we compute a family of fundamental solutions for the fractional Dirac operator. Finally, we point out that for the particular case of $\alpha=\beta=\gamma=1$ the obtained formulas coincide with the correspondents classical formulas.

\section{Preliminaries}

\subsection{Fractional calculus and special functions}

Let $\left({ }^{C} D_{a^{+}}^{\alpha} f\right)(x)$ denote the Caputo fractional derivative of order $\alpha>0$ (see [10])

$$
\left({ }^{C} D_{a^{+}}^{\alpha} f\right)(x)=\frac{1}{\Gamma(n-\alpha)} \int_{a}^{x} \frac{f^{(n)}(t)}{(x-t)^{\alpha+1-n}} d t, \quad n=[\alpha]+1, \quad x>a,
$$

where $[\alpha]$ means the integer part of $\alpha$. When $0<\alpha<1$ then (1) takes the form

$$
\left({ }^{C} D_{a^{+}}^{\alpha} f\right)(x)=\frac{1}{\Gamma(1-\alpha)} \int_{a}^{x} \frac{f^{\prime}(t)}{(x-t)^{\alpha}} d t .
$$

The Riemann-Liouville fractional integral of order $\alpha>0$ is given by (see [10])

$$
\left(I_{a^{+}}^{\alpha} f\right)(x)=\frac{1}{\Gamma(\alpha)} \int_{a}^{x} \frac{f(t)}{(x-t)^{1-\alpha}} d t, \quad x>a .
$$

We denote by $I_{a^{+}}^{\alpha}\left(L_{1}\right)$ the class of functions $f$ represented by the fractional integral (3) of a summable function, that is $f=I_{a^{+}}^{\alpha} \varphi, \varphi \in L_{1}(a, b)$. A description of this class of functions was given in [13].

Theorem 2.1 A function $f \in I_{a^{+}}^{\alpha}\left(L_{1}\right), \alpha>0$ if and only if $I_{a^{+}}^{n-\alpha} f \in A C^{n}([a, b]), n=[\alpha]+1$ and $\left(I_{a^{+}}^{n-\alpha} f\right)^{(k)}(a)=$ $0, k=0, \ldots, n-1$.

In Theorem $2.1 A C^{n}([a, b])$ denotes the class of functions $f$, which are continuously differentiable on the segment $[a, b]$ up to order $n-1$ and $f^{(n-1)}$ is absolutely continuous on $[a, b]$. Removing the last condition in Theorem 2.1 we obtain the class of functions that admits a summable fractional derivative.

Definition 2.2 ([13]) A function $f \in L_{1}(a, b)$ has a summable fractional derivative $\left(D_{a^{+}}^{\alpha} f\right)(x)$ if $\left(I_{a^{+}}^{n-\alpha} f\right)(x) \in$ $A C^{n}([a, b])$, where $n=[\alpha]+1$.

If a function $f$ admits a summable fractional derivative, then the composition of (1) and (3) can be written in the form (see e.g. [12])

$$
\left(I_{a^{+}}^{\alpha}{ }^{C} D_{a^{+}}^{\alpha} f\right)(x)=f(x)-\sum_{k=0}^{n-1} \frac{f^{(k)}(a)}{k !}(x-a)^{k}, \quad n=[\alpha]+1 .
$$

If $f \in I_{a^{+}}^{\alpha}\left(L_{1}\right)$ then (4) reduces to $\left(I_{a^{+}}^{\alpha}{ }^{C} D_{a^{+}}^{\alpha} f\right)(x)=f(x)$. Nevertheless we note that $\left({ }^{C} D_{a^{+}}^{\alpha} I_{a^{+}}^{\alpha} f\right)(x)=f(x)$ in both cases. We observe that, in general, the semigroup property for the composition of Caputo fractional does not hold. We present three sufficient conditions under which the law of exponents hold. They can be applied in different situations accordingly with the conditions assumed to the function $f$.

Theorem 2.3 ( $[2, p .56])$ Let $f \in C^{k}[a, b], a>0$ and $k \in \mathbb{N}$. Moreover, let $\alpha, \beta>0$ be such that there exists $l \in \mathbb{N}$ with $l \leq k$ and $\alpha, \alpha+\beta \in[l-1, l]$. Then

$$
{ }^{C} D_{a^{+}}^{\alpha}{ }^{C} D_{a^{+}}^{\beta} f(x)={ }^{C} D_{a^{+}}^{\alpha+\beta} f(x) .
$$


This theorem highlights a constraint on the applicability of the semigroup both with respect to the request of smoothness of the function and with respect to the ranges of the real orders of differentiation $\alpha$ and $\beta$. This means, for example, that, if $\alpha \in(0,1]$, then the law of exponents is applicable if $\beta \in[0,1-\alpha)$ and $f \in C^{k}$, with $k=1$. Here we notice that in most cases the law of exponents is not applicable for fractional Caputo derivatives, but anyhow there are different techniques to handle sequential fractional derivatives (see for example [12]). Since for $f \in C^{[\alpha]+1}([a, b])$ the Caputo derivative is a special case of the Grünwald-Letnikov fractional derivative (see $[12, \S 2.2 .3]$ ) then we have the following theorem:

Theorem 2.4 ([12, § 2.2.6]) Let $\alpha, \beta>0$ and $f \in C^{n}([a, b]), a>0, n=[\alpha]+1$. Then

$$
{ }^{C} D_{a^{+}}^{\alpha}{ }^{C} D_{a^{+}}^{\beta} f(x)={ }^{C} D_{a^{+}}^{\alpha+\beta} f(x)
$$

holds for arbitrary $\beta$ if the function $f$ satisfies the conditions

$$
f^{(k)}(a)=0, \quad \text { for } \quad k=0,1, \ldots, n-2 .
$$

For functions $f(x)$ that have a locally integrable singularity at $x=a$ we have the following result.

Theorem 2.5 ([5]) Suppose that $f(x)=(x-a)^{\lambda} g(x)$, where $a, \lambda>0$ and $g(x)$ has the generalized power series expansion $g(x)=\sum_{n=0}^{\infty} a_{n}(x-a)^{n \gamma}$ with radius of convergence $R>0,0<\gamma \leq 1$. Then

$$
{ }^{C} D_{a^{+}}^{\alpha}{ }^{C} D_{a^{+}}^{\beta} f(x)={ }^{C} D_{a^{+}}^{\alpha+\beta} f(x)
$$

for all $(x-a) \in(0, R)$, the coefficients $a_{n}=0$ for $n$ given by $n \gamma+\lambda-\beta=0$ and either

(a) $\lambda>\mu, \mu=\max (\beta+[\alpha],[\beta+\alpha])$

or

(b) $\lambda \leq \mu, a_{k}=0$, for $k=0,1, \ldots,\left[\frac{\mu-\lambda}{\gamma}\right]$, here $[x]$ denotes the greatest integer less than or equal to $x$.

One important function used in this paper is the two-parameter Mittag-Leffler function $E_{\mu, \nu}(z)[6]$, which is defined in terms of the power series by

$$
E_{\mu, \nu}(z)=\sum_{n=0}^{\infty} \frac{z^{n}}{\Gamma(\mu n+\nu)}, \quad \mu>0, \nu \in \mathbb{R}, z \in \mathbb{C} .
$$

In particular, the function $E_{\mu, \nu}(z)$ is entire of order $\rho=\frac{1}{\mu}$ and type $\sigma=1$. The exponential, trigonometric and hyperbolic functions are expressed through (9) as follows (see [6]):

$$
\begin{gathered}
E_{1,1}(z)=e^{z}, \quad E_{2,1}\left(-z^{2}\right)=\cos (z), \quad E_{2,1}\left(z^{2}\right)=\cosh (z), \\
z E_{2,2}\left(-z^{2}\right)=\sin (z), \quad z E_{2,2}\left(z^{2}\right)=\sinh (z)
\end{gathered}
$$

Two important fractional integral and differential formulae involving the two-parametric Mittag-Leffler function are the following

$$
\begin{gathered}
I_{a^{+}}^{\alpha}\left((x-a)^{\nu-1} E_{\mu, \nu}\left(k(x-a)^{\mu}\right)\right)=(x-a)^{\alpha+\nu-1} E_{\mu, \nu+\alpha}\left(k(x-a)^{\mu}\right) \\
{ }^{C} D_{a^{+}}^{\alpha}\left((x-a)^{\nu-1} E_{\mu, \nu}\left(k(x-a)^{\mu}\right)\right)=(x-a)^{\nu-\alpha-1} E_{\mu, \nu-\alpha}\left(k(x-a)^{\mu}\right)
\end{gathered}
$$

for all $\alpha>0, \mu>0, \nu \in \mathbb{R}, k \in \mathbb{C}, a>0, x>a$.

Our approach leads to the resolution of a linear Abel integral equation of the second kind, which solution is given using the Mittag-Leffler function, accordingly with the next Theorem.

Theorem 2.6 ([6, Thm. 4.2]) Let $f \in L_{1}[a, b], \alpha>0$ and $\lambda \in \mathbb{C}$. Then the integral equation

$$
u(x)=f(x)+\frac{\lambda}{\Gamma(\alpha)} \int_{a}^{x}(x-t)^{\alpha-1} u(t) d t, \quad x \in[a, b]
$$

has a unique solution

$$
u(x)=f(x)+\lambda \int_{a}^{x}(x-t)^{\alpha-1} E_{\alpha, \alpha}\left(\lambda(x-t)^{\alpha}\right) f(t) d t
$$




\subsection{Clifford analysis}

Let $\left\{e_{1}, \cdots, e_{d}\right\}$ be the standard basis of the Euclidean vector space in $\mathbb{R}^{d}$. The associated Clifford algebra $\mathbb{R}_{0, d}$ is the free algebra generated by $\mathbb{R}^{d}$ modulo $x^{2}=-\|x\|^{2} e_{0}$, where $x \in \mathbb{R}^{d}$ and $e_{0}$ is the neutral element with respect to the multiplication operation in the Clifford algebra $\mathbb{R}_{0, d}$. The defining relation induces the multiplication rules

$$
e_{i} e_{j}+e_{j} e_{i}=-2 \delta_{i j},
$$

where $\delta_{i j}$ denotes the Kronecker's delta. In particular, $e_{i}^{2}=-1$ for all $i=1, \ldots, d$. The standard basis vectors thus operate as imaginary units. A vector space basis for $\mathbb{R}_{0, d}$ is given by the set $\left\{e_{A}: A \subseteq\{1, \ldots, d\}\right\}$ with $e_{A}=e_{l_{1}} e_{l_{2}} \ldots e_{l_{r}}$, where $1 \leq l_{1}<\ldots<l_{r} \leq d, 0 \leq r \leq d, e_{\emptyset}:=e_{0}:=1$. Each $a \in \mathbb{R}_{0, d}$ can be written in the form $a=\sum_{A} a_{A} e_{A}$, with $a_{A} \in \mathbb{R}$. The conjugation in the Clifford algebra $\mathbb{R}_{0, d}$ is defined by $\bar{a}=\sum_{A} a_{A} \bar{e}_{A}$, where $\bar{e}_{A}=\bar{e}_{l_{r}} \bar{e}_{l_{r-1}} \ldots \bar{e}_{l_{1}}$, and $\bar{e}_{j}=-e_{j}$ for $j=1, \ldots, d, \bar{e}_{0}=e_{0}=1$. An important subspace of the real Clifford algebra $\mathbb{R}_{0, d}$ is the so-called space of paravectors $\mathbb{R}_{1}^{d}=\mathbb{R} \bigoplus \mathbb{R}^{d}$, being the sum of scalars and vectors. Each non-zero vector $a \in \mathbb{R}_{1}^{d}$ has a multiplicative inverse given by $\frac{\bar{a}}{\|a\|^{2}}$.

A $\mathbb{R}_{0, d}$-valued function $f$ over $\Omega \subseteq \mathbb{R}_{1}^{d}$ has the representation $f=\sum_{A} e_{A} f_{A}$, with components $f_{A}: \Omega \rightarrow$ $\mathbb{R}_{0, d}$. Properties such as continuity or differentiability have to be understood componentwise. Next, we recall the Euclidean Dirac operator $D=\sum_{j=1}^{d} e_{j} \partial_{x_{j}}$, which factorizes the $d$-dimensional Euclidean Laplace, i.e., $D^{2}=-\Delta$. A $\mathbb{R}_{0, d}$-valued function $f$ is called left-monogenic if it satisfies $D u=0$ on $\Omega$ (resp. right-monogenic if it satisfies $u D=0$ on $\Omega$ ).

For more details about Clifford algebras and basic concepts of its associated function theory we refer the interested reader for example to [1].

\section{Method of separation of variables}

\subsection{Eigenfunctions and fundamental solution of the fractional Laplace operator}

We consider the eigenfunction problem for the fractional Laplace operator ${ }^{C} \Delta_{+}^{\alpha} u(x)=\lambda u(x)$, i.e.,

$$
\left({ }^{C} D_{x_{0}^{+}}^{1+\alpha} u\right)(x, y, z)+\left({ }^{C} D_{y_{0}^{+}}^{1+\beta} u\right)(x, y, z)+\left({ }^{C} D_{z_{0}^{+}}^{1+\gamma} u\right)(x, y, z)=\lambda u(x, y, z)
$$

where $\lambda \in \mathbb{C},(\alpha, \beta, \gamma) \in] 0,1]^{3},(x, y, z) \in \Omega=\left[x_{0}, X_{0}\right] \times\left[y_{0}, Y_{0}\right] \times\left[z_{0}, Z_{0}\right], x_{0}, y_{0}, z_{0}>0, X_{0}, Y_{0}, Z_{0}<\infty$, and $u(x, y, z)$ admits summable fractional derivatives ${ }^{C} D_{x_{0}^{+}}^{1+\alpha},{ }^{C} D_{y_{0}^{+}}^{1+\beta}$ and ${ }^{C} D_{z_{0}^{+}}^{1+\gamma}$. Taking the integral operator $I_{x_{0}^{+}}^{1+\alpha}$ from both sides of (14) and taking into account (4) we get

$$
\begin{aligned}
& u(x, y, z)-u\left(x_{0}, y, z\right)-\left(x-x_{0}\right) u_{x}^{\prime}\left(x_{0}, y, z\right)+\left(I_{x_{0}^{+}}^{1+\alpha C} D_{y_{0}^{+}}^{1+\beta} u\right)(x, y, z)+\left(I_{x_{0}^{+}}^{1+\alpha} C_{z_{0}^{+}}^{1+\gamma} u\right)(x, y, z) \\
& =\lambda\left(I_{x_{0}^{+}}^{1+\alpha} u\right)(x, y, z) .
\end{aligned}
$$

Now, applying the operator $I_{y_{0}^{+}}^{1+\beta}$ to both sides of the previous expression and using Fubini's Theorem we get

$$
\begin{aligned}
& \left(I_{y_{0}^{+}}^{1+\beta} u\right)(x, y, z)-\left(I_{y_{0}^{+}}^{1+\beta} f_{0}\right)(y, z)-\left(x-x_{0}\right)\left(I_{y_{0}^{+}}^{1+\beta} f_{1}\right)(y, z)+\left(I_{x_{0}^{+}}^{1+\alpha} u\right)(x, y, z) \\
& -\left(I_{x_{0}^{+}}^{1+\alpha} u\right)\left(x, y_{0}, z\right)-\left(y-y_{0}\right)\left(I_{x_{0}^{+}}^{1+\alpha} u_{y}^{\prime}\right)\left(x, y_{0}, z\right)+\left(I_{x_{0}^{+}}^{1+\alpha} I_{y_{0}^{+}}^{1+\beta} C_{z_{0}^{+}}^{1+\gamma} u\right)(x, y, z) \\
& =\lambda\left(I_{x_{0}^{+}}^{1+\alpha} I_{y_{0}^{+}}^{1+\beta} u\right)(x, y, z),
\end{aligned}
$$

where we denote the Cauchy's fractional integral conditions by

$$
f_{0}(y, z)=u\left(x_{0}, y, z\right), \quad f_{1}(y, z)=u_{x}^{\prime}\left(x_{0}, y, z\right) .
$$

Finally, applying the operator $I_{z_{0}^{+}}^{1+\gamma}$ to both sides of equation (16) and using Fubini's Theorem we get

$$
\begin{aligned}
& \left(I_{y_{0}^{+}}^{1+\beta} I_{z_{0}^{+}}^{1+\gamma} u\right)(x, y, z)-\left(I_{y_{0}^{+}}^{1+\beta} I_{z_{0}^{+}}^{1+\gamma} f_{0}\right)(y, z)-\left(x-x_{0}\right)\left(I_{y_{0}^{+}}^{1+\beta} I_{z_{0}^{+}}^{1+\gamma} f_{1}\right)(y, z) \\
& \quad+\left(I_{x_{0}^{+}}^{1+\alpha} I_{z_{0}^{+}}^{1+\gamma} u\right)(x, y, z)-\left(I_{x_{0}^{+}}^{1+\alpha} I_{z_{0}^{+}}^{1+\gamma} h_{0}\right)(x, z)-\left(y-y_{0}\right)\left(I_{x_{0}^{+}}^{1+\alpha} I_{z_{0}^{+}}^{1+\gamma} h_{1}\right)(x, z) \\
& \quad+\left(I_{x_{0}^{+}}^{1+\alpha} I_{y_{0}^{+}}^{1+\beta} u\right)(x, y, z)-\left(I_{x_{0}^{+}}^{1+\alpha} I_{y_{0}^{+}}^{1+\beta} u\right)\left(x, y, z_{0}\right)-\left(z-z_{0}\right)\left(I_{x_{0}^{+}}^{1+\alpha} I_{y_{0}^{+}}^{1+\beta} u_{z}^{\prime}\right)\left(x, y, z_{0}\right) \\
& =\lambda\left(I_{x_{0}^{+}}^{1+\alpha} I_{y_{0}^{+}}^{1+\beta} I_{z_{0}^{+}}^{1+\gamma} u\right)(x, y, z)
\end{aligned}
$$


which is equivalent to

$$
\begin{aligned}
& \left(I_{y_{0}^{+}}^{1+\beta} I_{z_{0}^{+}}^{1+\gamma} u\right)(x, y, z)+\left(I_{x_{0}^{+}}^{1+\alpha} I_{z_{0}^{+}}^{1+\gamma} u\right)(x, y, z)+\left(I_{x_{0}^{+}}^{1+\alpha} I_{y_{0}^{+}}^{1+\beta} u\right)(x, y, z)-\lambda\left(I_{x_{0}^{+}}^{1+\alpha} I_{y_{0}^{+}}^{1+\beta} I_{z_{0}^{+}}^{1+\gamma} u\right)(x, y, z) \\
& =\left(I_{y_{0}^{+}}^{1+\beta} I_{z_{0}^{+}}^{1+\gamma} f_{0}\right)(y, z)+\left(x-x_{0}\right)\left(I_{y_{0}^{+}}^{1+\beta} I_{z_{0}^{+}}^{1+\gamma} f_{1}\right)(y, z) \\
& +\left(I_{x_{0}^{+}}^{1+\alpha} I_{z_{0}^{+}}^{1+\gamma} h_{0}\right)(x, z)+\left(y-y_{0}\right)\left(I_{x_{0}^{+}}^{1+\alpha} I_{z_{0}^{+}}^{1+\gamma} h_{1}\right)(x, z) \\
& \quad+\left(I_{x_{0}^{+}}^{1+\alpha} I_{y_{0}^{+}}^{1+\beta} g_{0}\right)(x, y)+\left(z-z_{0}\right)\left(I_{x_{0}^{+}}^{1+\alpha} I_{y_{0}^{+}}^{1+\beta} g_{1}\right)(x, y)
\end{aligned}
$$

where we denote the Cauchy's fractional integral conditions by

$$
\begin{aligned}
h_{0}(x, z) & =u\left(x, y_{0}, z\right), & h_{1}(x, z) & =u_{y}^{\prime}\left(x, y_{0}, z\right), \\
g_{0}(x, y) & =u\left(x, y, z_{0}\right), & g_{1}(x, y) & =u_{z}^{\prime}\left(x, y, z_{0}\right) .
\end{aligned}
$$

Assume that the eigenfunctions are such that $u(x, y, z)=u_{1}(x) u_{2}(y) u_{3}(z)$. Substituting in (18) and taking into account the initial conditions (17), (19), and (20) we obtain

$$
\begin{aligned}
& u_{1}(x)\left(I_{y_{0}^{+}}^{1+\beta} u_{2}(y) I_{z_{0}^{+}}^{1+\gamma} u_{3}(z)\right)+u_{2}(y)\left(I_{x_{0}^{+}}^{1+\alpha} u_{1}(x) I_{z_{0}^{+}}^{1+\gamma} u_{3}(z)\right) \\
& \quad+u_{3}(z)\left(I_{x_{0}^{+}}^{1+\alpha} u_{1}(x) I_{y_{0}^{+}}^{1+\beta} u_{2}(y)\right)-\lambda\left(I_{x_{0}^{+}}^{1+\alpha} u_{1}\right)(x)\left(I_{y_{0}^{+}}^{1+\beta} u_{2}\right)(y)\left(I_{z_{0}^{+}}^{1+\gamma} u_{3}\right)(z) \\
& =a_{1}\left(I_{y_{0}^{+}}^{1+\beta} u_{2}(y) I_{z_{0}^{+}}^{1+\gamma} u_{3}(z)\right)+a_{2}\left(x-x_{0}\right)\left(I_{y_{0}^{+}}^{1+\beta} u_{2}(y) I_{z_{0}^{+}}^{1+\gamma} u_{3}(z)\right) \\
& \quad+b_{1}\left(I_{x_{0}^{+}}^{1+\alpha} u_{1}(x) I_{z_{0}^{+}}^{1+\gamma} u_{3}(z)\right)+b_{2}\left(y-y_{0}\right)\left(I_{x_{0}^{+}}^{1+\alpha} u_{1}(x) I_{z_{0}^{+}}^{1+\gamma} u_{3}(z)\right) \\
& \quad+c_{1}\left(I_{x_{0}^{+}}^{1+\alpha} u_{1}(x) I_{y_{0}^{+}}^{1+\beta} u_{2}(y)\right)+c_{2}\left(z-z_{0}\right)\left(I_{x_{0}^{+}}^{1+\alpha} u_{1}(x) I_{y_{0}^{+}}^{1+\beta} u_{2}(y)\right)
\end{aligned}
$$

where

$$
a_{1}=u_{1}\left(x_{0}\right), \quad a_{2}=u_{1, x}^{\prime}\left(x_{0}\right), \quad b_{1}=u_{2}\left(y_{0}\right), \quad b_{2}=u_{2, y}^{\prime}\left(y_{0}\right), \quad c_{1}=u_{3}\left(z_{0}\right), \quad c_{2}=u_{3, z}^{\prime}\left(z_{0}\right)
$$

are constants defined by the initial conditions (17), (19), and (20). Supposing that

$$
\left(I_{x_{0}^{+}}^{1+\alpha} u_{1}\right)(x)\left(I_{y_{0}^{+}}^{1+\beta} u_{2}\right)(y)\left(I_{z_{0}^{+}}^{1+\gamma} u_{3}\right)(z) \neq 0,
$$

for $(x, y, z) \in \Omega$, we can divide (21) by this factor. Separating the variables we get the following three Abel's type second kind integral equations:

$$
\begin{aligned}
& u_{1}(x)-\mu\left(I_{x_{0}^{+}}^{1+\alpha} u_{1}\right)(x)=a_{1}+a_{2}\left(x-x_{0}\right), \\
& u_{2}(y)+\nu\left(I_{y_{0}^{+}}^{1+\beta} u_{2}\right)(y)=b_{1}+b_{2}\left(y-y_{0}\right), \\
& u_{3}(z)+(\mu-\lambda-\nu)\left(I_{z_{0}^{+}}^{1+\gamma} u_{3}\right)(z)=c_{1}+c_{2}\left(z-z_{0}\right),
\end{aligned}
$$

where $\lambda, \mu, \nu \in \mathbb{C}$ are constants. We observe that the equality

$$
\left(I_{x_{0}^{+}}^{1+\alpha} u_{1}\right)(x)\left(I_{y_{0}^{+}}^{1+\beta} u_{2}\right)(y)\left(I_{z_{0}^{+}}^{1+\gamma} u_{3}\right)(z)=0
$$

for at least one point $\left(x^{*}, y^{*}, z^{*}\right)$ agrees with (21), (22), (23), and (24). Solving the latter equations using (12) in Theorem 2.6 and after straightforward computations we obtain the following family of eigenfunctions $u_{\lambda, \mu, \nu}(x, y, z)=u_{1}(x) u_{2}(y) u_{3}(z)$, with

$$
\begin{aligned}
& u_{1}(x)=a_{1} E_{1+\alpha, 1}\left(\mu\left(x-x_{0}\right)^{1+\alpha}\right)+a_{2}\left(x-x_{0}\right) E_{1+\alpha, 2}\left(\mu\left(x-x_{0}\right)^{1+\alpha}\right), \\
& u_{2}(y)=b_{1} E_{1+\beta, 1}\left(-\nu\left(y-y_{0}\right)^{1+\beta}\right)+b_{2}\left(y-y_{0}\right) E_{1+\beta, 2}\left(-\nu\left(y-y_{0}\right)^{1+\beta}\right), \\
& u_{3}(z)=c_{1} E_{1+\gamma, 1}\left((\mu-\lambda-\nu)\left(z-z_{0}\right)^{1+\gamma}\right)+c_{2}\left(z-z_{0}\right) E_{1+\gamma, 2}\left((\mu-\lambda-\nu)\left(z-z_{0}\right)^{1+\gamma}\right) .
\end{aligned}
$$

For the particular case of $\lambda=0$ (fundamental solution), $\mu=2, \nu=1, x_{0}=y_{0}=z_{0}=0, X_{0}=Z_{0}=5, Y_{0}=15$, and $a_{i}=b_{i}=c_{i}=1$, with $i=1,2$, we show the graphical representation of the components $u_{1}, u_{2}, u_{3}$ for $\alpha, \beta$, $\gamma$ equal to $\frac{1}{4}, \frac{1}{2}, \frac{3}{4}, 1$. The plots were obtained using the software Mathematica 9 since this software is able to evaluate and to graphically represent functions involving the Mittag-Leffler functions. 

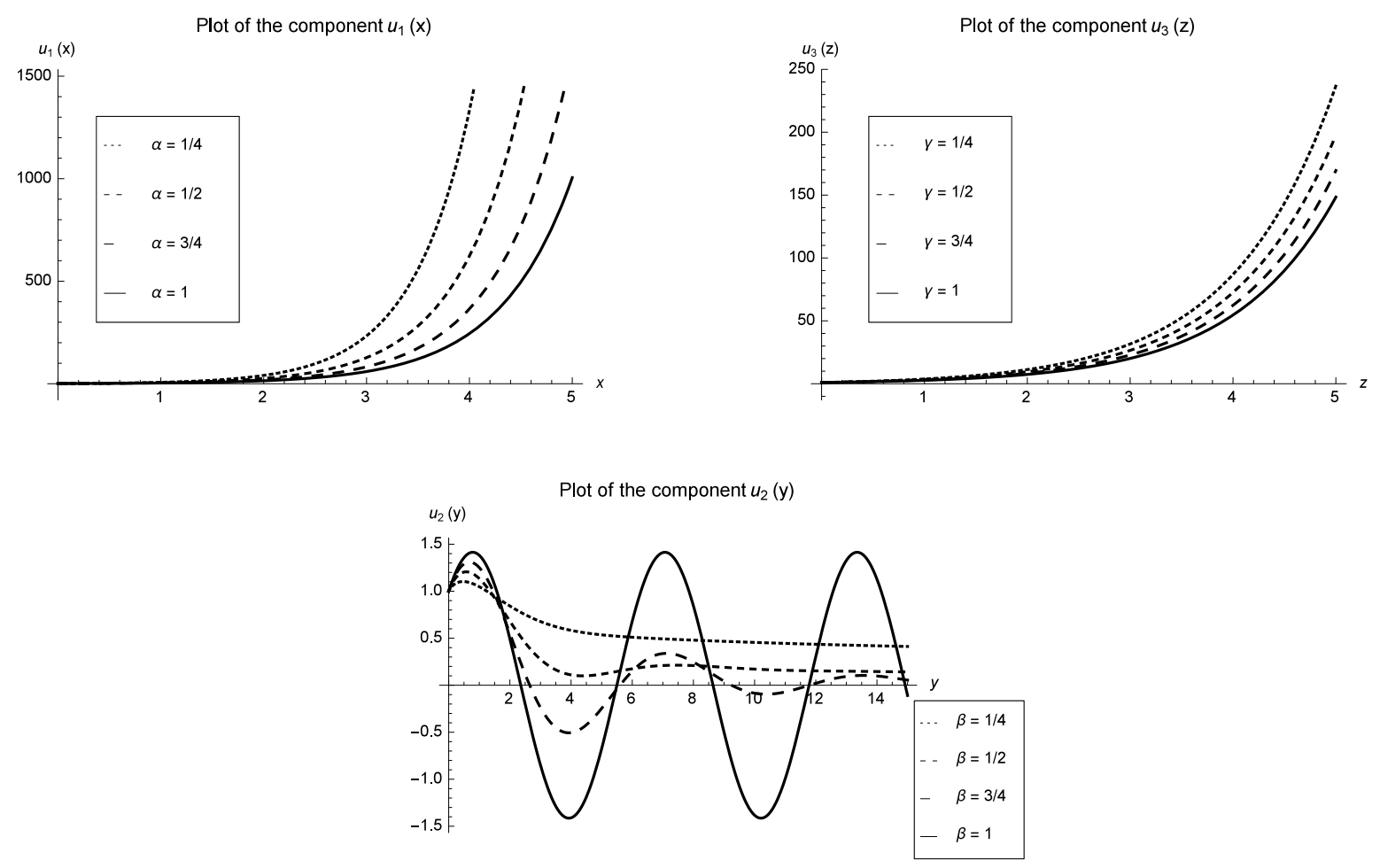

Figure 1: Plots of the components $u_{1}, u_{2}$ and $u_{3}$, when $\lambda=0, \mu=2, \nu=1, x_{0}=y_{0}=z_{0}=0, X_{0}=Z_{0}=5$, $Y_{0}=15$, and $a_{i}=b_{i}=c_{i}=1$, and different values of $\alpha, \beta$, and $\gamma$.

From the plots we observe that the components $u_{1}$ and $u_{3}$ are of exponential type and the increasing of the curve coincides with the decreasing of the parameters. For the component $u_{2}$ the sinusoidal behavior observed in the classical case $\beta=1$ suffers a relaxation with the decreasing of the parameter $\beta$.

Remark 3.1 In the special case of $\alpha=\beta=\gamma=1$ the functions $u_{1}, u_{2}$ and $u_{3}$ take the form

$$
\begin{aligned}
& u_{1}(x)=a_{1} \cosh \left(\sqrt{\mu}\left(x-x_{0}\right)\right)+\frac{a_{2}}{\sqrt{\mu}} \sinh \left(\sqrt{\mu}\left(x-x_{0}\right)\right), \\
& u_{2}(y)=b_{1} \cos \left(\sqrt{\nu}\left(y-y_{0}\right)\right)+\frac{b_{2}}{\sqrt{\nu}} \sin \left(\sqrt{\nu}\left(y-y_{0}\right)\right), \\
& u_{3}(z)=c_{1} \cosh \left(\sqrt{\mu-\lambda-\nu}\left(z-z_{0}\right)\right)+\frac{c_{2}}{\sqrt{\mu-\lambda-\nu}} \sinh \left(\sqrt{\mu-\lambda-\nu}\left(z-z_{0}\right)\right),
\end{aligned}
$$

which are the components of the eigenfunctions of the Laplace operator in $\mathbb{R}^{3}$ obtained by the method of separation of variables.

\subsection{Fundamental solution of the fractional Dirac operator}

In this section we compute the fundamental solution for the three dimensional fractional left Dirac operator defined via Caputo derivatives

$$
\left.\left.{ }^{C} D_{+}^{(\alpha, \beta, \gamma)}:=e_{1}{ }^{C} D_{x_{0}^{+}}^{\frac{1+\gamma}{2}}+e_{2}{ }^{C} D_{y_{0}^{+}}^{\frac{1+\beta}{2}}+e_{3}{ }^{C} D_{z_{0}^{+}}^{\frac{1+\gamma}{2}}, \quad(\alpha, \beta, \gamma) \in\right] 0,1\right]^{3} .
$$

This operator factorizes the fractional Laplace operator ${ }^{C} \Delta_{+}^{(\alpha, \beta, \gamma)}$ for Clifford valued functions $f$ given by $f(x, y, z)=\sum_{A} e_{A} f_{A}(x, y, z)$, where $e_{A} \in\left\{1, e_{1}, e_{2}, e_{3}, e_{1} e_{2}, e_{1} e_{3}, e_{2} e_{3}, e_{1} e_{2} e_{3}\right\}$, and each real valued function $f_{A}$ satisfies one of the sufficient conditions presented in Theorems 2.3, 2.4 or 2.5. In fact, for such functions we can apply the semigroup property (5) to obtain

${ }^{C} D_{x_{0}^{+}}^{\frac{1+\gamma}{2}}\left({ }^{C} D_{x_{0}^{+}}^{\frac{1+\gamma}{2}} f_{A}\right)={ }^{C} D_{x_{0}^{+}}^{1+\alpha} f_{A}, \quad{ }^{C} D_{y_{0}^{+}}^{\frac{1+\beta}{2}}\left({ }^{C} D_{y_{0}^{+}}^{\frac{1+\beta}{2}} f_{A}\right)={ }^{C} D_{y_{0}^{+}}^{1+\beta} f_{A}, \quad{ }^{C} D_{z_{0}^{+}}^{\frac{1+\gamma}{2}}\left({ }^{C} D_{z_{0}^{+}}^{\frac{1+\gamma}{2}} f_{A}\right)={ }^{C} D_{z_{0}^{+}}^{1+\gamma} f_{A}$. 
Moreover, for the mixed fractional derivatives ${ }^{C} D_{x_{0}^{+}}^{\frac{1+\gamma}{2}}\left({ }^{C} D_{y_{0}^{+}}^{\frac{1+\beta}{2}} f_{A}\right)$, due to the Leibniz's rule for the differentiation under integral sign, Fubini's Theorem and Schwarz's Theorem, we have

$$
\begin{aligned}
& { }^{C} D_{x_{0}^{+}}^{\frac{1+\gamma}{2}}\left({ }^{C} D_{y_{0}^{+}}^{\frac{1+\beta}{2}} f_{A}\right)={ }^{C} D_{y_{0}^{+}}^{\frac{1+\beta}{2}}\left({ }^{C} D_{x_{0}^{+}}^{\frac{1+\gamma}{2}} f_{A}\right), \\
& { }^{C} D_{x_{0}^{+}}^{\frac{1+\gamma}{2}}\left({ }^{C} D_{z_{0}^{+}}^{\frac{1+\gamma}{2}} f_{A}\right)={ }^{C} D_{z_{0}^{+}}^{\frac{1+\gamma}{2}}\left({ }^{C} D_{x_{0}^{+}}^{\frac{1+\gamma}{2}} f_{A}\right), \\
& { }^{C} D_{y_{0}^{+}}^{\frac{1+\beta}{2}}\left({ }^{C} D_{z_{0}^{+}}^{\frac{1+\gamma}{2}} f_{A}\right)={ }^{C} D_{z_{0}^{+}}^{\frac{1+\gamma}{2}}\left({ }^{C} D_{y_{0}^{+}}^{\frac{1+\beta}{2}} f_{A}\right) .
\end{aligned}
$$

From (32), (33), (34), (35) and the multiplication rules (13) of the Clifford algebra, we finally get

$$
{ }^{C} D_{+}^{(\alpha, \beta, \gamma)}\left({ }^{C} D_{+}^{(\alpha, \beta, \gamma)} f\right)=-{ }^{C} \Delta_{+}^{(\alpha, \beta, \gamma)} f
$$

i.e., the fractional Dirac operator factorizes the fractional Laplace operator.

In order to get the fundamental solution of ${ }^{C} D_{+}^{(\alpha, \beta, \gamma)}$ we apply this operator to the fundamental solution $u(x, y, z)=u_{1}(x) u_{2}(y) u_{3}(z)$, where $u_{i}$ are given by (25), (26) and (27), respectively . To make the calculations we make use of the derivation rule (11) and the fractional analogous formula for differentiation of integrals depending on a parameter where the upper limit also depends on the same parameter (see [12, Section 2.7.4]). Hence,

$$
\begin{aligned}
U(x, y, z) & =\left({ }^{C} D_{+}^{(\alpha, \beta, \gamma)} u\right)(x, y, z) \\
& =e_{1} u_{2}(y) u_{3}(z)\left({ }^{C} D_{x_{0}^{+}}^{\frac{1+\gamma}{2}} u_{1}\right)(x)+e_{2} u_{1}(x) u_{3}(z)\left({ }^{C} D_{y_{0}^{+}}^{\frac{1+\beta}{2}} u_{2}\right)(y)+e_{3} u_{1}(x) u_{2}(y)\left({ }^{C} D_{z_{0}^{+}}^{\frac{1+\gamma}{2}} u_{3}\right)(z)
\end{aligned}
$$

where $u_{1}, u_{2}, u_{3}$ are given respectively by (25), (26), (27) and

$$
\begin{aligned}
\left({ }^{C} D_{x_{0}^{+}}^{\frac{1+\gamma}{2}} u_{1}\right)(x)= & a_{1}\left(x-x_{0}\right)^{-\frac{1+\alpha}{2}} E_{1+\alpha, \frac{1-\alpha}{2}}\left(\mu\left(x-x_{0}\right)^{1+\alpha}\right)+a_{2}\left(x-x_{0}\right)^{\frac{1-\alpha}{2}} E_{1+\alpha, \frac{3-\alpha}{2}}\left(\mu\left(x-x_{0}\right)^{1+\alpha}\right) \\
\left({ }^{C} D_{y_{0}^{+}}^{\frac{1+\beta}{2}} u_{2}\right)(y)= & b_{1}\left(y-y_{0}\right)^{-\frac{1-\beta}{2}} E_{1+\beta, \frac{1-\beta}{2}}\left(-\nu\left(y-y_{0}\right)^{1+\beta}\right)+b_{2}\left(y-y_{0}\right)^{\frac{1-\beta}{2}} E_{1+\beta, \frac{3-\beta}{2}}\left(-\nu\left(y-y_{0}\right)^{1+\beta}\right) \\
\left({ }^{C} D_{z_{0}^{+}}^{\frac{1+\gamma}{2}} u_{3}\right)(z)= & c_{1}\left(z-z_{0}\right)^{\frac{1-\gamma}{2}} E_{1+\gamma, \frac{1-\gamma}{2}}\left((-\mu+\lambda+\nu)\left(z-z_{0}\right)^{1+\gamma}\right) \\
& +c_{2}\left(z-z_{0}\right)^{\frac{1-\gamma}{2}} E_{1+\gamma, \frac{3-\gamma}{2}}\left((-\mu+\lambda+\nu)\left(z-z_{0}\right)^{1+\gamma}\right) .
\end{aligned}
$$

Remark 3.2 In the special case of $\alpha=\beta=\gamma=1, u_{1}, u_{2}$ and $u_{3}$ take the form (28), (29) and (30), respectively, and expressions (38), (39), and (40) take the form

$$
\begin{aligned}
& \left(D_{x} u_{1}\right)(x)=a_{1} \sqrt{\mu} \sinh \left(\sqrt{\mu}\left(x-x_{0}\right)\right)+a_{2} \sinh \left(\sqrt{\mu}\left(x-x_{0}\right)\right), \\
& \left(D_{y} u_{2}\right)(y)=b_{1} \sqrt{\nu} \sin \left(\sqrt{\nu}\left(y-y_{0}\right)\right)+b_{2} \cos \left(\sqrt{\nu}\left(y-y_{0}\right)\right), \\
& \left(D_{z} u_{3}\right)(z)=c_{1} \sqrt{-\mu+\lambda+\nu} \sinh \left(\sqrt{\mu-\lambda-\nu}\left(z-z_{0}\right)\right)+c_{2} \sinh \left(\sqrt{\mu-\lambda-\nu}\left(z-z_{0}\right)\right),
\end{aligned}
$$

which are the components of the fundamental solution of the Dirac operator in $\mathbb{R}^{3}$ obtained by the method of separation of variables.

Acknowledgement: The authors were supported by Portuguese funds through the CIDMA - Center for Research and Development in Mathematics and Applications, and the Portuguese Foundation for Science and Technology ("FCT-Fundação para a Ciência e a Tecnologia"), within project UID/MAT/ 0416/2013. N. Vieira was also supported by FCT via the FCT Researcher Program 2014 (Ref: IF/00271/2014). 


\section{References}

[1] R. Delanghe, F. Sommen and V. Souček, Clifford algebras and spinor-valued functions. A function theory for the Dirac operator, Mathematics and its Applications-Vol.53, Kluwer Academic Publishers, Dordrecht, 1992.

[2] K. Diethelm, The analysis of fractional differential equations. An application-oriented exposition using differential operators of Caputo type, Lecture Notes in Mathematics, Springer, Berlin, 2004.

[3] M. Ferreira and N. Vieira, Eigenfunctions and fundamental solutions of the fractional Laplace and Dirac operators: the Riemman-Liouville case, Complex Anal. Oper. Theory, 10-No.5, (2016), 1081-1100.

[4] M. Ferreira and N. Vieira, Eigenfunctions and fundamental solutions of the fractional Laplace and Dirac operators using Caputo derivatives, submitted.

[5] M. Garg and P. Manohar, Three-dimensional generalized differential transform method for space-time fractional diffusion equation in two space variables with variable coefficients, Palest. J. Math., 4-No.1, (2015), 127-135.

[6] R. Gorenflo, A.A. Kilbas, F. Mainardi and S. Rogosin, Mittag-Leffler functions. Theory and applications, Springer Monographs in Mathematics, Springer, Berlin, 2014.

[7] R. Gorenflo, Y. Luchko and M. Stojanovć, Fundamental solution of a distributed order time-fractional diffusion-wave equation as probability density, Fract. Calc. Appl. Anal., 16-No.2, (2013), 297-316.

[8] R. Gorenflo and F. Mainardi, Fractional calculus: integral and differential equations of fractional order, In: Fractals and Fractional Calculus in Continuum Mechanics, Eds: A. Carpinteri and F. Mainardi, Springer Verlag, Wien-New York, (1997), 223-276.

[9] B. Guo, X. Pu and F. Huang, Fractional partial differential equations and their numerical solutions, World Scientific, Hackensack, NJ, 2015.

[10] A. Kilbas, H.M. Srivastava and J.J. Trujillo, Theory and applications of fractional differential equations, NorthHolland Mathematics Studies-Vol.204, Elsevier, Amsterdam, 2006.

[11] S.K. Miller and B. Ross, An introduction to the fractional calculus and fractional differential equations, John Wiley \& Sons Inco., New York, 1993.

[12] I. Podlubny, Fractional differential equations. An introduction to fractional derivatives, fractional differential equations, to methods of their solution and some of their applications, Mathematics in Science and Engineering-Vol.198, Academic Press, San Diego, CA, 1999.

[13] S.G. Samko, A.A. Kilbas and O.I. Marichev, Fractional integrals and derivatives: theory and aqpllications, Gordon and Breach, New York, NY, 1993.

[14] J.L. Vázquez, Recent progress in the theory of nonlinear diffusion with fractional Laplacian operators, Discrete Contin. Dyn. Syst., Ser.7-No.4, (2014), 857-885.

[15] S. Yakubovich, Eigenfunctions and fundamental solutions of the fractional two-parameter Laplacian, Int. J. Math. Math. Sci., Article ID 541934, (2010), 18p.

[16] Y. Zhou, Basic theory of fractional differential equations, World Scientific, Hackensack, NJ, 2014. 